\title{
4
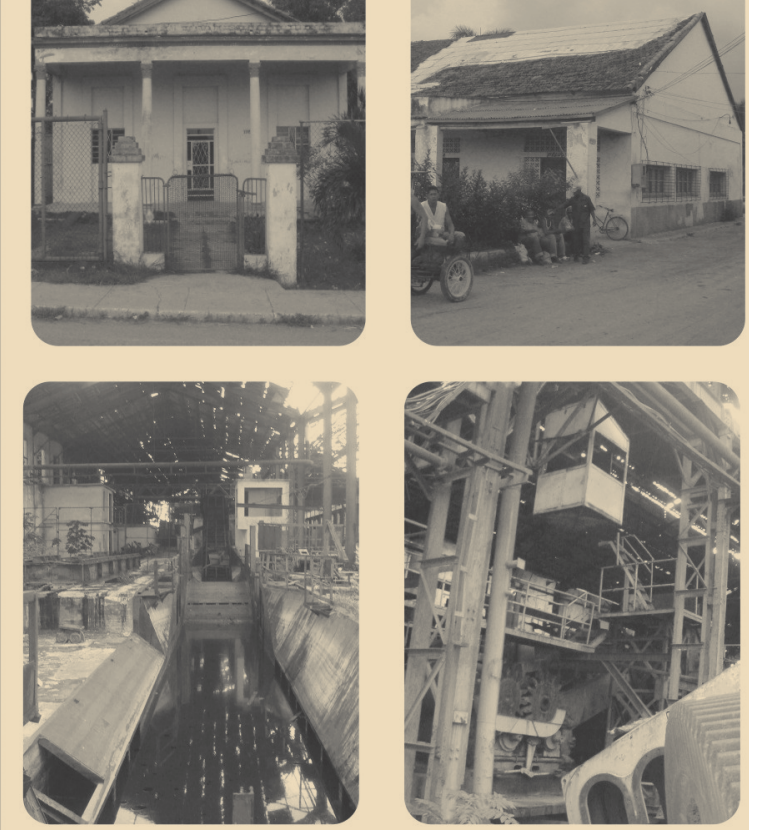 \\ Guía para el diagnóstico de conjuntos industriales azucareros: el caso del central Toledo, La Habana, Cuba
}
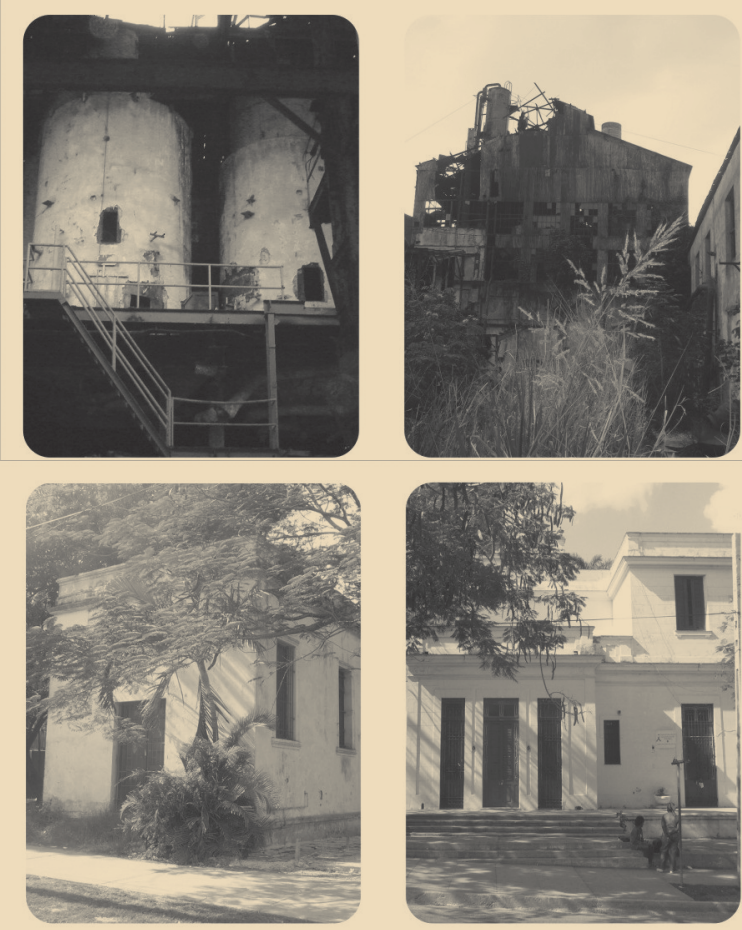

Guidelines for the Diagnosis of Industrial Sugar Mills: The Case of Central Toledo, La Habana, Cuba

\section{Adrián González González}

Universidad Tecnológica de La Habana "José Antonio Echeverría" (CUJAE), Cuba yoadrianxxi@gmail.com

\section{Indira Costa Fallarero}

Université Paris-Sorbone I, Francia

icostafall@gmail.com

\section{Tania Gutiérrez Rodríguez}

Universidad Tecnológica de La Habana "José Antonio Echeverría" (CUJAE), Cuba taniarq1946@gmail.com

\section{Resumen}

Los centrales, o ingenios, azucareros tienen estrecha relación con el desarrollo histórico y cultural de Cuba: al contener valores, tangibles e intangibles, estos conjuntos se han vuelto parte vital del patrimonio y la identidad nacionales. El redimensionamiento de la industria azucarera en el 2000 puso en peligro la salvaguardia de su patrimonio histórico construido: si bien no todo puede conservarse, sí deben respetarse algunas piezas y ciertas manifestaciones indispensables. El objeto de esta INVESTIGACIÓN es organizar los criterios para diagnosticar tales conjuntos desactivados, en coherencia con sus valores. Se presenta el diseño de un instrumento que guía este proceso, en el cual los valores se consideran como el principal factor de transformación y, con base en ello, se identifican potencialidades y restricciones para futuras propuestas de rehabilitación. Se muestran de manera sintética los resultados de la aplicación del instrumento en un caso peculiar, los cuales demuestran la conveniencia de utilizar el patrimonio industrial como elemento de cambio de dichos conjuntos.

\section{Palabras claves}

patrimonio industrial; central azucarero; ingenio azucarero; Cuba; guía; diagnóstico

\section{Abstract}

The Cuban sugar mills are closely related to the historical and cultural development of Cuba. Those settlements show values, both tangible and intangible, which are a vital part of the national heritage and identity. The downsizing of the Cuban sugar industry in year 2000 endangered the 
preservation of its historical heritage. Although not everything can be preserved, representative pieces must be respected. The purpose of this INVESTIGATION is to organize the criteria for the diagnosis of these industrial settlements, consistent with their values. This paper presents the design of an instrument that guides this process, in which the values are considered the main factor of these transformations. Based on this, the potentialities and constraints are identified for future rehabilitation proposals. Furthermore, the results of the application of the instrument are shown in summary for a particular case. These results demonstrate the convenience of using industrial heritage as a transformation factor for these settlements.

\section{Keywords}

industrial heritage; Cuban sugar mill; Cuba; guidelines; diagnosis

\section{Introducción: relevancia y estado de conservación del patrimonio industrial azucarero en Cuba}

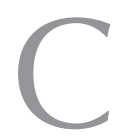
on la creación, en 1971, de la Sociedad para la Arqueología Industrial, y, posteriormente, en 1978, del International Committee for the Conservation of the Industrial Heritage $(\mathrm{TICCIH}$, Comité Internacional para la Conservación del Patrimonio Industrial), se sentaron las bases para el inventario, catalogación, protección y reconocimiento de los valores del patrimonio industrial a escala internacional (Ríos 2007:26). Asimismo, se dio origen al amplio concepto que actualmente se acepta para definir este tipo de patrimonio, el cual permite considerar todos los elementos, tangibles o intangibles, que de una forma $u$ otra se relacionan con la actividad de un complejo industrial (cfr. TICCIH 2003:art. 1). A la par, la Carta de Nizhny Tagil sobre el patrimonio industrial estableció que el valor de este tipo de bienes culturales puede ser de carácter histórico, tecnológico, social, arquitectónico o científico (cfr. TICCIH 2003:art. 1).

En Cuba, hacer referencia a su actividad fabril significa hablar de la industria azucarera. Tras la Revolución haitiana, en 1791-1803, Cuba se convirtió en la primera productora de azúcar mundial, condición que consolidó con una industria de monocultivo que la insertó en el mercado internacional y la ligó indisolublemente con la historia y el desarrollo del país (Lozano 2009:1). El siglo XIX fue el periodo que marcó la consolidación de esta industria en Cuba, basada en la introducción de adelantos tecnológicos, como la máquina de vapor, en el año 1819, y la construcción, en 1873, del primer ferrocarril hispanoamericano (Lozano 2009:1). Las guerras de independencia cubanas marcaron un retroceso en su desarrollo, a consecuencia de las operaciones militares, lo que debilitó la base económica de ese sector (Lozano 2009:1). Finalizada la guerra contra España, y tras la intervención de Estados Unidos de América en la isla, la industria estaba expuesta a la penetración del capital extranjero, fundamentalmente, el norteamericano (Lozano 2009:1).

El siglo XX, con el establecimiento de la república, marcó el inicio de un movimiento extraordinario de creación de grandes fábricas de azúcar, que comenzó en 1900 en Las Villas, Camagüey y Oriente, para extenderse paulatinamente al resto del país; en 1958 Cuba se convirtió en el primer exportador de azúcar del mundo (Charadán 1982:115).

A partir de esta actividad se conformó, en lo social, lo económico y en el paisaje físico de las regiones donde se desarrolló, una identidad propia que responde a la producción del dulce (Pereira 2009:36). El central ${ }^{1}$ y su batey, ${ }^{2}$ expresión física fundamental de la industria azucarera en Cuba, configuró el territorio de la isla, se convirtió en la unidad económica fundamental del espacio donde se emplaza y derivó en tradiciones, sentimientos y modos de vida arraigados en códigos particulares, tal y como lo indica Barnet (2005:6):

El azúcar unió a Cuba. La cultura que se generó en su ámbito, conforma hoy la cultura nacional. El batey, coto cerrado, célula fundamental, contribuyó a la fusión integradora de todos los valores originarios de nuestro país [...] donde se dan el abrazo definitorio todas las manifestaciones que componen nuestro acervo espiritual y material.

Con el triunfo de la Revolución, en enero de 1959, principió la transformación socialista de la industria, marcada por la nacionalización de las empresas; en los años setenta se alcanzaron los rendimientos agrícolas cañeros más altos de la historia y se iniciaron procesos de unificación de la agricultura con la industria azucarera (Barnet 2005:6). Los factores políticos y económicos de la década de 1990 deprimieron sensiblemente todo el sistema productivo, y en 2002 se emprendió una reestructuración de la industria que condujo a la reducción del número de centrales para concentrar la producción y las tierras por cultivar en las más eficientes (Barnet 2005:6).

\footnotetext{
${ }^{1}$ Central: término para denominar en Cuba a las fábricas de azúcar. A partir del siglo xx, comenzó a sustituir el antiguo vocablo de ingenio, cuando la fábrica dejó de moler únicamente la caña producida en las plantaciones propias, y tras un proceso de concentración industrial, empezó a moler caña proveniente de plantaciones monofuncionales, conocidas como colonias cañeras (Dembicz 1989:14).

2 Batey: entre los aborígenes era la plaza donde jugaban sus batos, o pelotas; más tarde fue el espacio que ocupaban las fábricas, sus plazas o patios y recintos próximos a las haciendas de campo. El uso de este término ha trascendido aun hasta nuestros días; se utiliza para hacer referencia al conjunto de instalaciones que se erige alrededor del central en función de la actividad azucarera, incluidas las viviendas de los trabajadores y sus servicios. Coexiste con otros términos empleados como equivalentes, por ejemplo, comunidad, pueblo, poblado e, incluso, central, este último, visto como algo más que la fábrica de azúcar (Cruz 2009:45).
} 

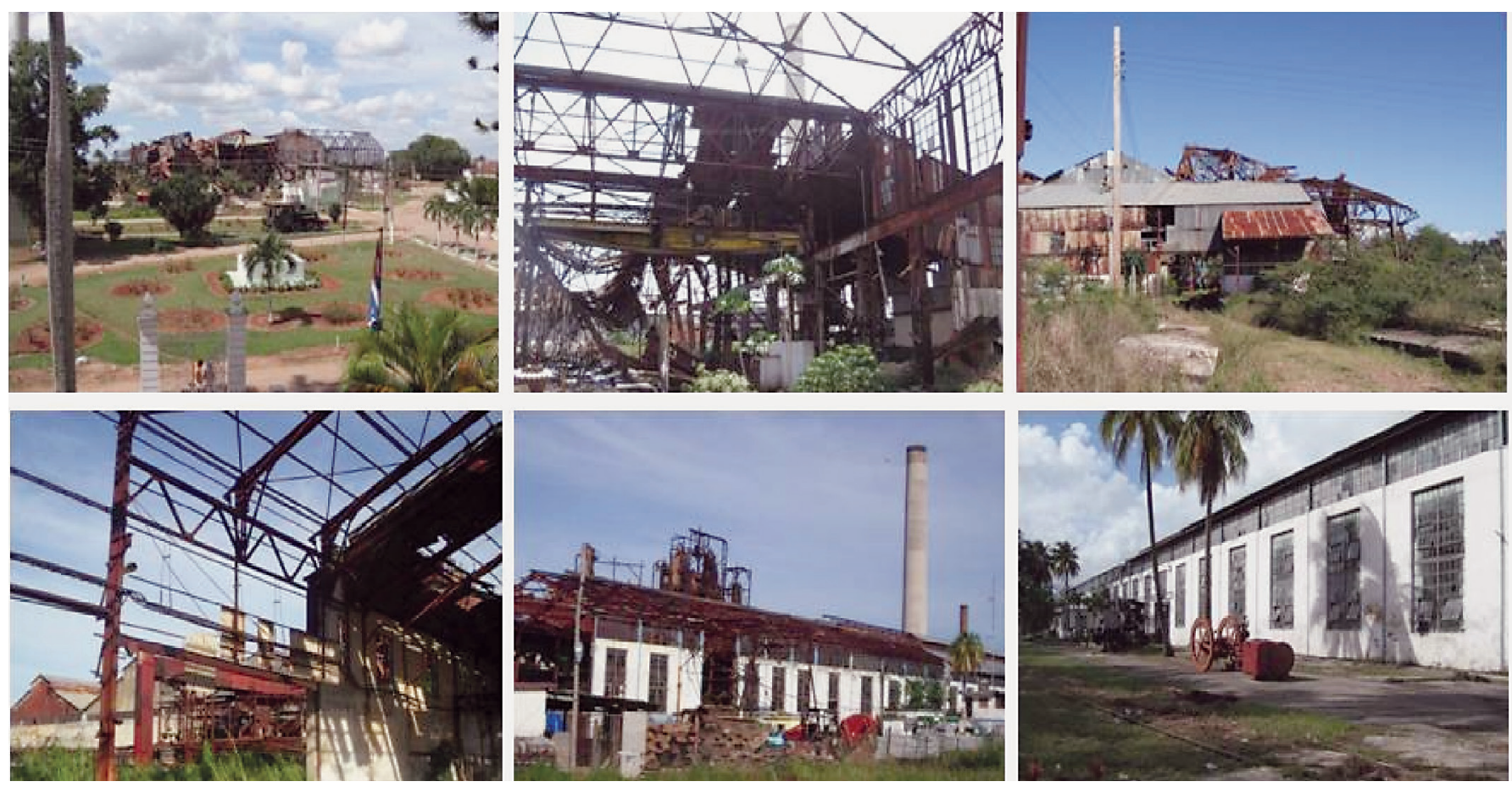

FIGURA 1. Aspecto del deterioro de instalaciones industriales desactivadas de los centrales Amistad, Hershey y Toledo (Fotografía: Adrián González González, Indira Costa Fallarero y Tania Gutiérrez Rodríguez, 2008).

No obstante, los bateyes azucareros han perdurado como monumentos del pasado cañero de Cuba. Ortiz (1983:44) ha abordado su complejidad en tanto patrimonio industrial: "el central no es una simple explotación agraria, ni siquiera una planta fabril [...] es todo un sistema de tierras, máquinas, transporte, técnicas, obreros, dineros y población para producir azúcar; es todo un organismo social, tan vivo y complejo como una ciudad". Siguiendo esta definición, nuestro estudio acometió tres componentes esenciales del patrimonio industrial azucarero cubano, a seguir:

En primer lugar, al central y su batey, ${ }^{3}$ unidad indisoluble que incluye el edificio productivo, el área habitacional con sus servicios y toda una amplia infraestructura técnica asociada con la producción de azúcar: almacenes, tanques de combustible, talleres de maquinado, de pailería, ${ }^{4}$ entre otros. En segundo término, las redes ferroviarias y todo el paisaje que se generó a partir del cultivo de la caña de azúcar y el uso del ferrocarril como medio

\footnotetext{
${ }^{3}$ No puede hacerse referencia al central y al batey de forma independiente: uno depende del otro y se influyen de manera recíproca e inequívoca. La organización del proceso productivo azucarero, que incluye desde las construcciones que éste requería, hasta las relaciones económico-sociales que generó, conforma y determina en gran medida la estructura y la morfología del batey. Para Rallo y Segre (s.f.:67), el batey refleja de modo acusado su función productora y la estructura de clases sociales que lo integraba.

${ }^{4}$ Especialidad profesional de la rama de fabricación mecánica que tiene como función principal la construcción con acero laminado de silos, cisternas y otros depósitos aptos para el almacenaje y transporte de líquidos, gases y sólidos en forma de granos (cfr. Rallo y Segre s.f.:67).
}

de transporte principal de esta industria. ${ }^{5}$ En tercer sitio, cuanto surgió y se desarrolló con base en la actividad productiva y que creó una forma de vida marcada por costumbres y tradiciones muy arraigadas, es decir, la cultura particular que produjo el batey, la cual ha sido elemento básico para la conformación de la conciencia nacional, la arquitectura, la geografía, la danza, la música e incluso la composición étnica de Cuba (Barnet 2005:12; Linares 2005:87).

Desafortunadamente, tanto la significación de esta "cultura del azúcar" para la formación de la nacionalidad cubana como el propio patrimonio industrial azucarero en Cuba padecen, cada vez más, una situación desfavorable para su conservación (Gutiérrez 2013:251). A pesar de los numerosos ejemplos en el mundo que demuestran la validez y conveniencia de reutilizar las fábricas y sus infraestructuras para lograr el desarrollo de los territorios (Capel 1996:29), en Cuba no se previeron las estrategias para transformar los espacios industriales de los conjuntos azucareros cuando se decidió su paralización, a finales del siglo xx. Esto, sin duda, conduce al riesgo actual de perder, de forma irreparable, exponentes significativos de un importante patrimonio cultural cubano. Conjuntos productivos como los centrales Hershey, Amistad o Toledo son ejemplos de esta realidad (Figura 1).

\footnotetext{
${ }^{5}$ A estos elementos, propios de todo central, deben sumarse los numerosos restos arqueológicos que aparecen por la vastedad del territorio, vestigios de formas de producción anteriores que, sin duda, constituyen antecesores del central azucarero.
} 
Las cifras son contundentes: de los más de 150 centrales que existían en el país en el año 2000, en la actualidad apenas funciona poco más de la tercera parte (Gutiérrez 2013:247). No sólo un centenar de fábricas de azúcar se paralizaron a lo largo de todo el país a principios del siglo XXI, sino que, en su mayoría, se desmantelaron y algunas se abandonaron a un futuro incierto (Gutiérrez 2008:3; Pell 2013:287).

Hoy en día el número de elementos por considerar cuando se hace referencia al patrimonio industrial azucarero cubano alude a centenares de exponentes dispersos por todo el territorio de la isla (Pell 2013:287). El desconocimiento sobre cuáles merecen reconocerse como valiosos y conservarse como tales es, desde nuestra perspectiva, una de las causas de la adversa situación del patrimonio azucarero cubano; de ahí que apremie un instrumento con el que diagnosticar estos conjuntos industriales para inventariar, catalogar y detectar sus valores $y$, con base en ello, tomar decisiones sobre su transformación.

\section{Fundamentos para la valoración del patrimonio industrial}

Nuestro estudio se fundamenta en la idea de que, en el difícil ejercicio de decidir qué es valioso dentro del patrimonio industrial, hay que evitar que el elemento patrimonial se convierta en un adorno o recuerdo; su concepto y tratamiento deben, más bien, favorecer que se considere un objeto de uso cotidiano que se disfruta no sólo por razón de su valor cultural sino también porque es útil. En concurrencia, desde hace años algunos expertos han insistido en que los inventarios del patrimonio histórico-artístico deben contener propuestas de transformación que impliquen su uso y funcionamiento (Capel 1996:39). Asimismo, Capel sostiene que el inventario y la catalogación han de hacerse con un fin y en un territorio determinados. Por ende, nuestra idea es que, en materia de conservación del patrimonio industrial, siempre hay que pensar en el futuro uso de los monumentos.

A partir de una revisión de las experiencias internacionales, vale afirmar que son diversas las opciones para la transformación en reutilización de los espacios industriales con valor patrimonial. ${ }^{6}$ Sin embargo, habrá que considerar que la aceptación de los nuevos usos implantados en los espacios en los que durante siglos se produjo azúcar está sujeta a la apropiación, uso y representatividad que la comunidad le confiera (Puig 2008:2-8). Asimismo, dado que el central constituye la unidad socioeconómica fundamental de la región donde está asentado, nuestra visión es que en cualquier intento de su refuncionalización será vital definir con claridad las necesidades de la comunidad

\footnotetext{
${ }^{6}$ Capel (1996:29-31) hace una apretada pero exhaustiva revisión de experiencias internacionales y reúne los ejemplos consultados en ocho grupos, demostrando la variedad de nuevos usos asumidos por los edificios industriales rehabilitados.
}

que se han de solucionar. Para ello es fundamental tener en cuenta los requerimientos de los potenciales usuarios, para satisfacerlos en la medida de lo posible y, así, lograr altos grados de apropiación y uso. Del mismo modo, para que la conservación de cada uno de los conjuntos industriales se desarrolle de forma sustentable ${ }^{7}$ es indispensable identificar sus potencialidades.

Fundado en lo anterior, nuestro estudio propone que los procesos de conservación y apropiación comiencen con la identificación y catalogación de los elementos del patrimonio industrial azucarero cubano y, subsecuentemente, continúen con la identificación de las necesidades y potencialidades que muestra el conjunto en las condiciones sociales actuales. Entendemos como potencialidades todo aquello que, a partir de los recursos locales del territorio, coadyuve tanto al desarrollo de nuevas funciones del bien patrimonial en escenarios futuros como a la satisfacción de las necesidades de la comunidad. El sistema de valores que se detecte en cada uno de estos conjuntos azucareros es parte imprescindible de ese grupo de potencialidades. Este sistema debe entenderse dentro de su contexto territorial para que desencadene un proceso de reutilización como vía para su conservación.

Con tales fundamentos, el objeto de esta INVESTIGACIÓN es organizar criterios y herramientas que lleven a realizar diagnósticos en conjuntos industriales azucareros en Cuba con el fin de detectar de manera holística aquellos elementos de valor presentes $y$, a la par, identificar las necesidades y potencialidades que estos bienes patrimoniales presentan para su reutilización. Nuestra misión, así, es vincular, sobre bases científicas, la conservación del patrimonio azucarero cubano con las nuevas formas de desarrollo local.

\section{Bases metodológicas: determinación analítica de criterios y herramientas de diagnóstico}

De acuerdo con Cárdenas (1993:53), al analizar las relaciones entre los significados atribuidos a un lugar y las variaciones en el uso en cuanto a frecuencia, intensidad y carácter de las actividades por realizar en él, se desvelan diferentes grados de apropiación, rechazo o indiferencia, dependiendo de cuánto se identifique la comunidad usuaria con los elementos significativos de ese espacio. Ello obedece a varios factores, todos explicados por esta misma autora (Cárdenas 1993:53), a seguir:

- En primer lugar, las características físicas del sitio y su capacidad general para dar cumplimiento a las actividades que deben realizarse en él.

\footnotetext{
${ }^{7}$ Capacidad que tiene la sociedad para hacer uso consciente y responsable de sus recursos — en este caso, del patrimonio-, sin exceder su capacidad de renovación y sin comprometer el acceso a éstos por parte de generaciones futuras (cfr. ONU 1987).
} 
- En segundo término, las particularidades de los sujetos que integran la comunidad usuaria.

- En tercer lugar, las relaciones sociales establecidas entre los miembros de esa comunidad.

- Como cuarto aspecto, el desarrollo global de la cultura ambiental, en particular, si se ha llamado la atención hacia los valores de un determinado entorno y si se ha generado una divulgación acerca de ellos.

Nuestra experiencia indica que las alternativas de transformación de los espacios industriales azucareros en Cuba - en particular, aquellas que tienen el propósito de lograr categorías de significación adecuadas, propiciar que los usuarios se identifiquen con ellos y, por lo tanto, favorecer un uso más pleno de los espacios como vía para conservar sus valores patrimoniales- están influidas fuertemente por las complejas relaciones entre los factores antes señalados. Esto obliga a que las propuestas de transformación de los espacios industriales se realicen con base en el conocimiento detallado de las características de la comunidad usuaria, de sus necesidades, valores e intereses, aspectos que deben incorporarse en el proceso de toma de decisiones de manera que el diseño y la ejecución consideren la participación activa de los agentes involucrados. Para ello también se hace necesario tanto generar una cultura del ambiente capaz de propiciar que esa participación sea efectiva como lograr un proceso de diseño y materialización de las propuestas que esté en contacto directo con el ambiente que se va a transformar.

Ahora bien, con la finalidad de comprender estas relaciones en los conjuntos azucareros cubanos, nuestro estudio determinó cuatro unidades de análisis que integran los diferentes campos de acción y de conocimiento del caso investigado, a saber:

- La imagen industrial, concebida, al ser contenedora de los valores patrimoniales por conservar, como el objeto de estudio.

- El contexto, entendido como el espacio que complementa la imagen industrial, donde se desarrollan sus relaciones económicas, político-ideológicas, sociales, físico-ambientales y tecnológicas, es decir, los factores culturales que determinan las necesidades y potencialidades en función de las cuales actuar.

- La rehabilitación, que constituye la herramienta para lograr la transformación, dependiendo de la conservación de los valores patrimoniales.

- La imaginabilidad se presenta como resultado del proceso de percepción mediante el cual el usuario reconoce el espacio, y, por lo tanto, como el mecanismo de apropiación que condiciona el grado de utilización.

La determinación de estas unidades de análisis ayuda a definir la información que caracteriza el funciona- miento del sistema central-batey, ya que para cada una de ellas establece variables de análisis específicas:

a) Las variables identificadas como correspondientes a la unidad imagen industrial se consideran como elementos que intervienen en la identificación, justamente, del carácter industrial del conjunto en cuestión. Para su estimación, se realiza un análisis íntegro de la función arquitectónica industrial, que se matiza por medio de los elementos que definen dicho carácter y, para terminar, se hace una relación detallada de las variables principales que definen los elementos que identifican la función fabril en un determinado inmueble.

b) Las condiciones que determinan la unidad contexto son aquellas que resultan relevantes en la identificación de las necesidades de la comunidad que requieren espacios construidos, así como recursos materiales y humanos locales, para dar respuesta a las mismas. En la revisión de los elementos que se han de tener presentes para analizar un contexto, Cárdenas (1998:189) y Fernández Figueroa (2007:58) consideran dos dimensiones: la social y la física. Nuestro estudio observó una más: la arquitectónica, ya que ésta permite tener en cuenta la capacidad del edificio industrial de atender las necesidades de la comunidad.

c) Para caracterizar la unidad rehabilitación se precisan las variables con las que determinar la realidad urbana por transformar, asociadas en tres grupos definidos como estructuras que reúnen las relaciones formales, funcionales y de significación que se producen en el contexto. Tomar en consideración estos elementos objetivos y tangibles junto a los subjetivos e intangibles permite, según Cazanave Macías (2007:30), ponderar o disminuir el abismo entre el espacio físico sobre el cual pueden actuar los profesionales y el espacio limitado por la percepción de la comunidad usuaria.

d) Por último, como variables de la unidad imaginabilidad se asumen los elementos definidos por Lynch: sendas, bordes, zonas, nodos e hitos (1970:47-49) para el análisis de la imagen urbana, así como los criterios postulados por López (1982:65) para estudiar la correspondencia entre los componentes de la ciudad y los de los espacios arquitectónicos.

La definición de las variables en cada una de las unidades descritas anteriormente se realizó a partir de la revisión bibliográfica y de conversaciones libres con personas representativas de los actores del sistema, cuyos resultados se caracterizaron como variables iniciales que posteriormente se validaron mediante la consulta, por entrevista, a expertos. En síntesis, el proceso se organizó en tres etapas.

En la primera se enumeraron y definieron las variables que determinan el sistema estudiado y su entorno. 


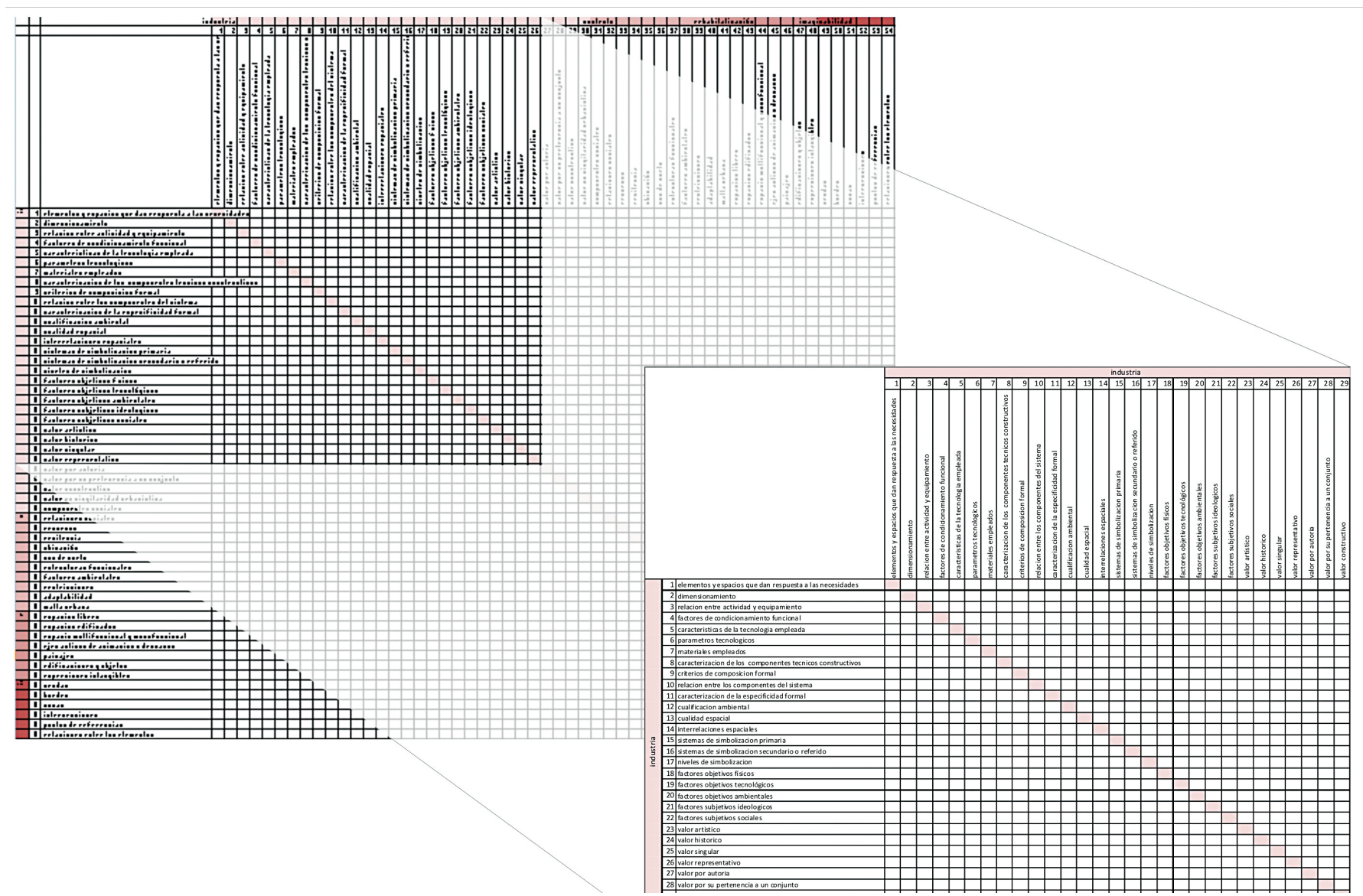

FIGURA 2. Esquema de las variables organizadas en matrices de impacto cruzado/MIC-MAC (Esquema: Adrián González González, Indira Costa FaIlarero y Tania Gutiérrez Rodríguez, 2009).

En la segunda etapa se analizaron, desde un enfoque sistémico, las relaciones entre aquellas variables, las cuales, para tal fin, se diagramaron en un modelo de matrices de impacto cruzado (Figura 2). Estas matrices dan la posibilidad de analizar las relaciones de influencia y dependencia entre las variables, de modo tal que organizan una reflexión sobre la estructura del sistema que, a su vez, permite obtener conclusiones sobre el papel de cada variable en el comportamiento de éste, con el objeto de identificar las que resultan relevantes (Cazanave 2007:37).

En tercer término, se definió la importancia de ciertas variables que juegan un papel principal en el sistema. ${ }^{8}$

En total, en el estudio se determinaron 54 variables estructuradoras, las que componen la base de la guía para el diagnóstico de conjuntos industriales azucareros cubanos que se explica a continuación.

\footnotetext{
${ }^{8}$ En esta última etapa se logró sólo un primer acercamiento al ordenamiento sistémico de las variables que describen el problema, cuyo principal valor está en el reajuste de aquellas definidas como principales. Sin embargo, el análisis total de variables debe abordarse con mayor detalle en futuras investigaciones.
}

\section{Guía para el diagnóstico de conjuntos industriales azucareros cubanos}

El desglose metodológico en variables explicado anteriormente favoreció la confección de una guía como instrumento capaz de orientar el diagnóstico de los conjuntos industriales azucareros en Cuba, la cual representa la principal contribución de nuestro estudio. El proceso metodológico de concepción de la guía se muestra de manera esquemática en la Figura 3.

La guía abarca todas las escalas de análisis y establece un orden de preponderancia entre los elementos que se han de diagnosticar según los niveles de influencia dentro del sistema. Para una mejor comprensión, la estructura se despliega de lo general a lo particular en estudios específicos: sobre el territorio, el contexto inmediato y el edificio industrial, que en cada caso incluye análisis de los antecedentes históricos, evolución, desarrollo y situación actual. Dentro de los parámetros que conforman el instrumento están implícitas las variables y sus interrelaciones definidas en las unidades de análisis ya presentadas.

En su concepción, la guía integra la identificación de los valores del espacio industrial y su diagnóstico desde un 


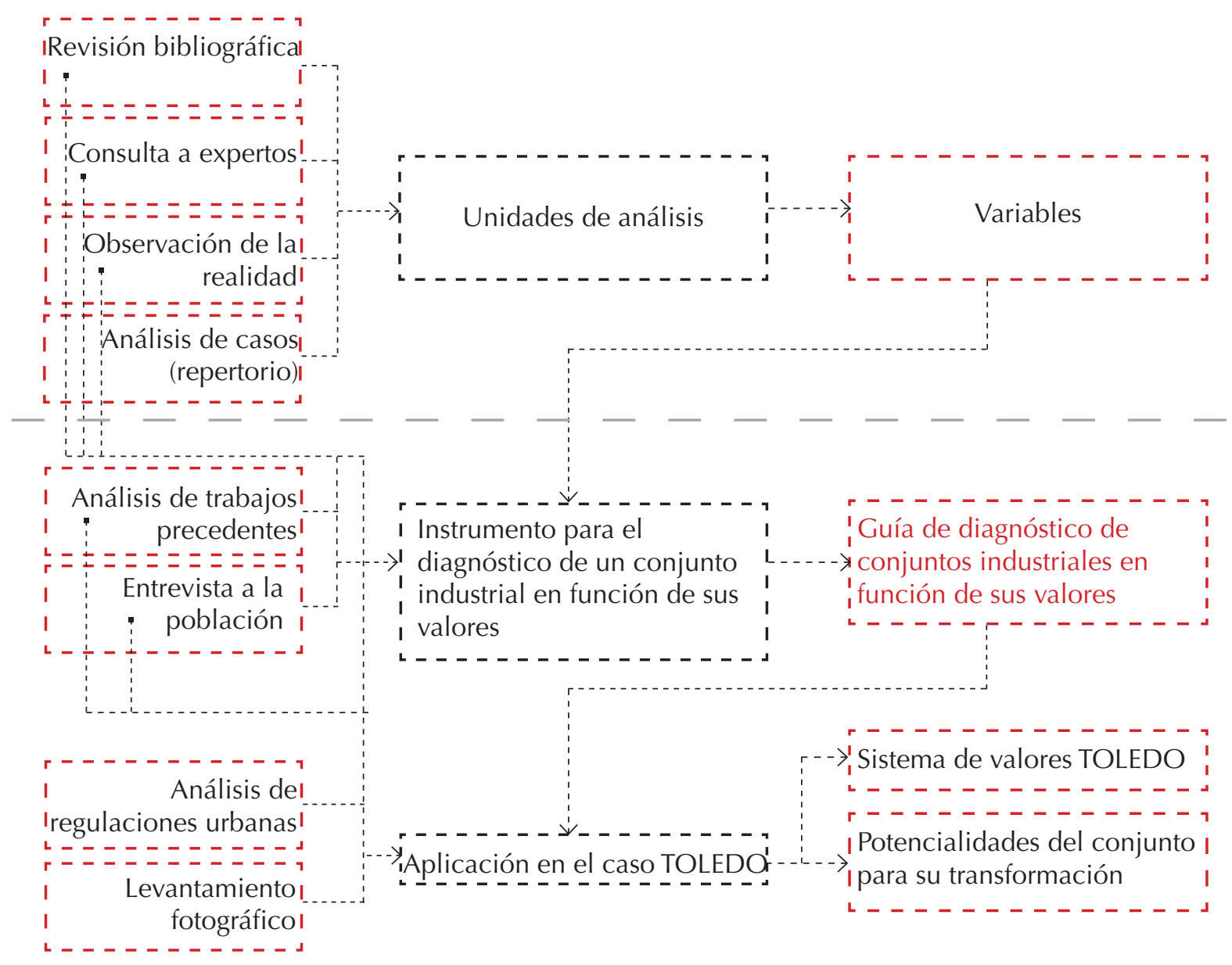

FIGURA 3. Esquema metodológico para la concepción de la guía (Esquema: Adrián González González, Indira Costa Fallarero y Tania Gutiérrez Rodríguez, 2009).

enfoque que pone especial énfasis en las características específicas del tema arquitectónico industrial. Así, se relaciona con la arquitectura y las infraestructuras técnica y social desde una perspectiva sistémica, lo que ayuda a determinar los elementos de valor.

La puesta en práctica de la guía busca aportar un inventario de todo el universo de elementos, tangibles e intangibles, que deben catalogarse para dictaminar la conveniencia de su conservación o transformación. Para facilitar la catalogación, y como complemento de la guía de diagnóstico, se requiere realizar investigación en ocho categorías que definen el tipo de valor, según la naturaleza del elemento en cuestión: valor artístico, ${ }^{9}$ histórico, ${ }^{10}$ valor por la singularidad urbanística o del proyecto ar-

\footnotetext{
${ }^{9}$ Valor artístico: se refiere a los elementos representativos de un estilo arquitectónico, poseedores de calidades de estilo, composición, materiales, coherencia tipológica y cualquier otra particularidad relevante (Ríos 2007:121-123).

${ }^{10}$ Valor histórico: alude a aquellos elementos que son testigos de una organización social o económica determinada y que ayudan a configurar la memoria histórica colectiva (Ríos 2007:121-123).
}

quitectónico de un edificio, ${ }^{11}$ por representatividad, ${ }^{12}$ autoría, ${ }^{13}$ pertenencia a un conjunto ${ }^{14}$ y valor constructivo o estructural. ${ }^{15}$ Estas categorías, además, deben analizarse en diferentes ámbitos territoriales: local, municipal,

\footnotetext{
${ }^{11}$ Valor por la singularidad: hace referencia a las características irreproducibles o de calidad única, ya del edificio o del diseño urbano, relacionadas tanto con aspectos del proyecto como con los técnico-constructivos (Ríos 2007:121-123).

${ }^{12}$ Valor por representatividad: versa sobre los elementos que son muestra de un modelo frecuente, como sucede en el caso de tipologías constructivas que se repiten por su pertenencia a una empresa determinada o una época (Ríos 2007:121-123).

${ }^{13}$ Valor por la autoría: habla de aquellos elementos que pertenezcan a un autor de reconocido prestigio y trayectoria profesional (Ríos 2007:121-123).

${ }^{14}$ Valor por pertenecer a un conjunto: apunta a elementos que pertenecen a un conjunto y contribuyen a dar coherencia tanto en un ámbito rural como en uno urbano; fundamentalmente, aquellos que permiten comprender el proceso productivo integralmente (Ríos 2007:121-123).

${ }^{15}$ Valor constructivo: se relaciona con los materiales utilizados en la construcción de un inmueble, de una tecnología determinada, de un proceso constructivo, etcétera (Ríos 2007:121-123).
} 
provincial, regional, nacional e internacional, con lo que se estima si el elemento tiene valor para la comunidad del conjunto o trasciende a una escala territorial mayor. Finalmente, los elementos por conservar pueden organizarse y expresarse en un sistema de valores.

En la guía se incluyen elementos que se refieren a la situación actual del conjunto industrial, lo que permite identificar los problemas que presenta el asentamiento; a partir de ello, las necesidades a resolver pueden establecerse en una propuesta de conservación, a la vez que se documentan las amenazas que se han de tomar en cuenta en la intervención. La guía también tiene el objeto de contribuir a organizar los elementos necesarios para definir las potencialidades del lugar para su rehabilitación.

El proceso de aplicación de la guía se organiza en tres pasos para cada uno de los elementos recogidos en el instrumento: 1) descripción, 2) identificación de valores y 3 ) valoración de oportunidades y fortalezas. En cada paso se sintetizan los resultados parciales obtenidos, lo que constituye un punto de referencia para el próximo, evitando la repetición de información.

En el primer paso, la descripción, se recoge la caracterización del elemento en cuestión. Dicha descripción debe ser lo más completa posible, pues conforma el primer inventario de los bienes, y una omisión en este momento podría conducir a que se ignore algún bien. Se le debe prestar atención a aquellas cuestiones que determinen el carácter productivo del asentamiento.

A partir de la descripción se produce la identificación de valores, o catalogación, seleccionando aquellos elementos que aportan valor. Para esto se deben usar como referencia los ocho criterios de valor definidos anteriormente. Es pertinente aclarar que un bien puede presentar más de un tipo de valor, lo cual debe considerarse a la hora de ponderar qué se conserva y qué no.

Por último, en el tercer momento se identifican aqueIlos otros elementos que, sin ser valiosos desde el punto de vista patrimonial cultural, muestran algún valor de uso, y representan recursos para la reconversión y reutilización del conjunto. Ellos se definen como oportunidades y fortalezas. Los resultados obtenidos en los pasos dos y tres se consideran como las potencialidades del conjunto para su conservación a partir de su reutilización.

\section{Aplicación del instrumento en el estudio del central Toledo}

El proceso descrito anteriormente se aplicó en el central Toledo, conocido actualmente como Martínez Prieto (Figura 4). El conjunto se ubica en el municipio Marianao, provincia de La Habana, próximo a la avenida 114, uno de los principales corredores urbanos de la capital cubana. Dentro del conjunto de centrales desactivados en Cuba, se trata de un caso particular, fundamentalmente, a causa de dos razones: por un lado, es el central más antiguo que siguió funcionando como tal hasta nuestros días en el mismo emplazamiento, de modo que es testimonio de las diferentes etapas tecnológicas por las que pasó la industria azucarera, y, por el otro, el crecimiento urbano de La Habana lo ha convertido en el único dentro de la ciudad, así como uno de los pocos centrales cubanos inmersos en un contexto urbano, a escasos metros de la Universidad Tecnológica de La Habana Cujae, la principal de tipo técnico de Cuba (Gutiérrez 2013:244, 260).

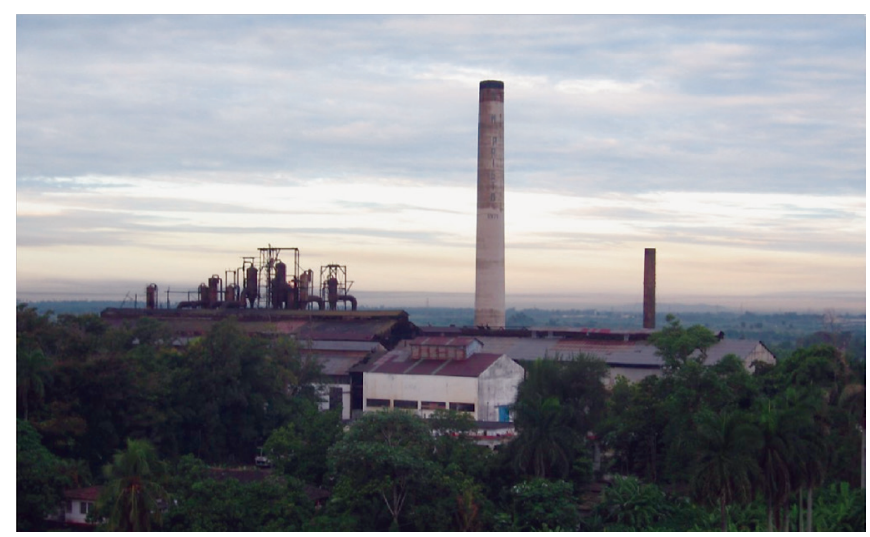

FIGURA 4. Central Toledo, aspecto de la industria antes del proceso de desmantelamiento (Fotografía: Adrián González González, Indira Costa Fallarero y Tania Gutiérrez Rodríguez, 2009).

La paralización del central Toledo comenzó a finales de la década de 1990, y se prolongó durante toda la primera década del siglo XXI, debido a diferentes decisiones respecto de nuevas funciones (refinería de azúcar crudo, empaquetadora de minidosis de azúcar refino y una propuesta de conversión en museo que no llegó a materializarse), terminando finalmente por ser objeto de un total desmantelamiento (Costa 2009:85).

Todo lo anterior atentó de forma agresiva contra la imagen histórica del conjunto y desencadenó una merma evidente de sus principales valores patrimoniales tangibles; comienzan a perderse exponentes valiosos, como las ruinas del barracón, la estructura morfológica tradicional característica de los bateyes del siglo XIX, la infraestructura ferroviaria, la casa del propietario, la capiIla y la fachada principal de la instalación industrial visible desde el asentamiento habitacional. El proceso de deterioro patrimonial ha estado aparejado a uno de degradación de la comunidad asociada al edificio productivo, el cual ha estado marcado por fuertes movimientos migratorios, pérdida de los servicios básicos de la comunidad, envejecimiento de la población, pérdida de la especialización en el trabajo y la cultura productiva acumulados en siglos de producción azucarera, entre otros (Gutiérrez 2013:260).

En términos formales, éste es un conjunto que, por su ubicación geográfica y dimensiones, relativamente pequeñas, de acuerdo con Gutiérrez (2013:261), está en condiciones de asimilar nuevas funciones adecuadas a las condiciones existentes y previsibles. 
La suma de estas circunstancias convierte al central Toledo en un caso especial y apto para la implementación de la guía propuesta. El procedimiento seguido para la aplicación de la guía en él puede explicarse a partir del proceso seguido con el elemento estructura activa, pues permite, al mismo tiempo, caracterizar brevemente el funcionamiento del batey en la actualidad y contribuir al entendimiento del conjunto. Los resultados obtenidos en cada uno de los tres pasos llevados a cabo: descripción, identificación de valores, y valoración de oportunidades y fortalezas, se hacen coincidir con cada una de las columnas que se muestran en la Figura 5.

En primer lugar, se describió la estructura activa del central Toledo, la cual está integrada por el sistema de es- pacios donde se desarrolla el sentido de tolerancia, identidad y respeto mutuo de la comunidad. Como resultado de este primer paso se definió que dicha estructura del batey está conformada por un gran espacio habitacional y dos puntos de concentración de servicios, donde se producen fundamentalmente todas las relaciones sociales. Destaca que la instalación industrial, la cual durante siglos fue el principal elemento aglutinador de éstas, actualmente no representa un elemento activo dentro de la estructura. La ciudad universitaria se considera como otro espacio activo del contexto inmediato.

La articulación de estos espacios se produce mediante un eje principal, que coincide con el fundamental identificado en la estructura morfológica, convirtiéndose en el
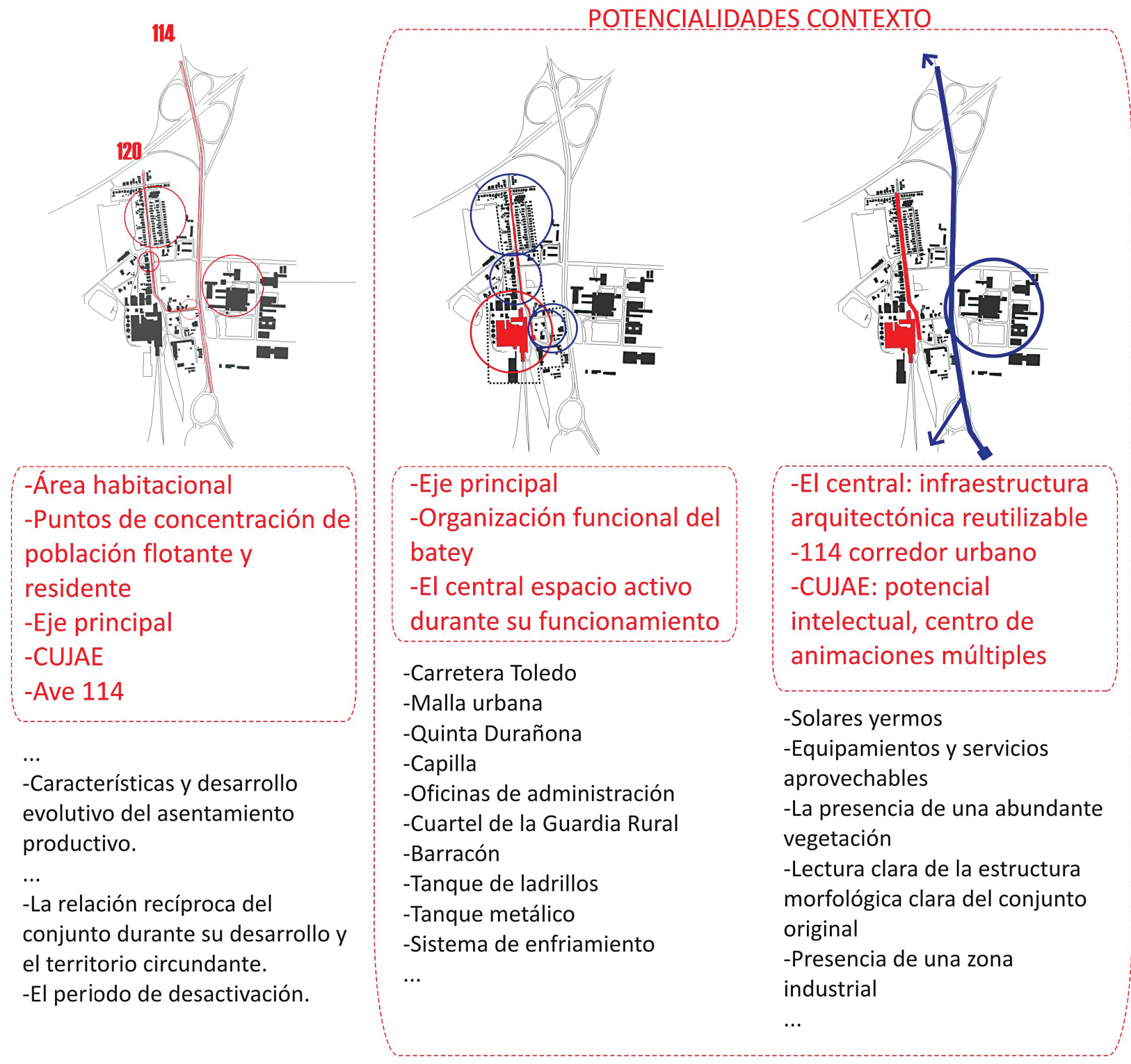

FIGURA 5. Aplicación de la guía en el caso de Toledo; se muestran los tres pasos del estudio para una de las variables indicadas en la guía: la estructura activa (Esquema: Adrian González González, Indira Costa Fallarero y Tania Gutiérrez Rodríguez, 2009). 
eje activo del conjunto más importante. El formado por la avenida 114 constituye no sólo el límite entre la comunidad y la ciudad universitaria sino, a la vez, la principal conexión de la zona con la ciudad, por lo que también forma parte de la estructura activa del conjunto.

A partir de esta descripción se seleccionaron los elementos que mostraban rasgos relevantes para alguna de las ocho categorías de valor definidas: la estructura urbana del batey, que muestra claramente la estructuración funcional tradicional; el área industrial, los servicios y los diferentes espacios residenciales; destacadamente, el eje principal del trazado urbano del batey como elemento articulador. Al mismo tiempo se escogieron otros elementos que pertenecen a la estructura activa, como la casa del dueño, el mercado, la capilla y las oficinas originales de la administración del central.

En un tercer momento se identificaron, asimismo, elementos que ofrecen oportunidades para la transformación del conjunto, entre los que resaltan: la universidad, el corredor urbano 114, y las instalaciones y espacios industriales en desuso.

Siguiendo este procedimiento se analizó cada uno de los elementos recogidos en el instrumento propuesto. Resultó notable en este conjunto: su emplazamiento, la jerarquización del eje vial principal como espacio lineal de organización urbana, la estructura morfológica del batey tradicional, la red ferroviaria, la infraestructura técnica, los restos del edificio industrial apreciables desde la comunidad, la presencia de grandes espacios en desuso condicionados por la demolición de los edificios productivos principales, la casa del propietario, la capilla, oficinas de administración y las ruinas de un barracón que en algún momento albergó esclavos (Figura 6).

El resultado fundamental de la aplicación de la guía fue, precisamente, la identificación de las potencialidades del conjunto, dentro de las cuales sobresale su sistema de valores como el principal elemento potencial para actuar en función de las necesidades igualmente detectadas con el instrumento. A partir de ello, también pueden definirse otras potencialidades, a seguir:

- La presencia de un legado, transmitido a través de generaciones de una población que no ha dejado de ostentar un elevado sentido de pertenencia. Sus estrechas relaciones de convivencia favorecen un trabajo comunitario para un mayor desarrollo y bienestar social.

- La estructura espacial del central, aún reconocible tras el desmantelamiento de las estructuras principales, la cual convoca a la inserción de nuevas funciones.

- La cercanía de una universidad, cuyo capital intelectual puede aportar fuerza laboral para intereses de desarrollo perspectivo.
- La trama urbana, que permite una rápida accesibilidad, interconexiones y una fácil orientación y lectura del conjunto, con identificación de los puntos focales.

- Las características ambientales de la zona, que propician el desarrollo de soluciones ecológicas.

- El predominio de la vegetación, que facilita la creación de lugares de esparcimiento y participación social con confort visual y climático.

- La presencia de reservas de terreno, que pueden servir para satisfacer necesidades habitacionales por crecimiento poblacional.

La aplicación de la guía en este conjunto constituyó la primera validación de ésta como un instrumento útil para el diagnóstico de los conjuntos industriales azucareros cubanos.

\section{Conclusiones}

Este estudio presentó resultados específicos y generales, sobre los cuales versan las presentes conclusiones.

En cuanto a los primeros, los alcanzados con la puesta en práctica de la guía en el caso de estudio del central Toledo demostraron el carácter global y sistémico del análisis realizado, ya que éste abarcó la evolución en el tiempo de los elementos constitutivos y valorativos, así como, en paralelo, analizó la influencia de la industria en las diferentes escalas: territorio, contexto y arquitectura. Adicionalmente, el empleo de la metodología evidenció la relevancia del elemento industrial en el territorio, a la par que reveló la necesidad y la conveniencia de la reconversión del espacio industrial como principal vía para la consolidación del conjunto urbano. El estudio también develó las particularidades del contexto y fue posible determinar la influencia que ejercen los elementos del sitio en la posible transformación del espacio industrial. En particular, resaltan algunos resultados, tales como la definición de un sistema de valores que posteriormente se recoge en un catálogo fácilmente generalizable; se creó de un plano de potencialidades y se conformó una estrategia de intervención, ambos, útiles como base para futuras acciones en el conjunto. Asimismo, debe subrayarse que el proceso llevó a la identificación de una amplia gama de recursos con los que se cuenta para la creación de una propuesta coherente para el sitio.

En términos de las aportaciones generales, vale señalar que para la preservación del patrimonio industrial azucarero cubano no existen formas o criterios únicos; sin embargo, debemos ser capaces de identificar los componentes valiosos. Como no todo tiene valor, ni todo puede conservarse, la elaboración de una guía de diagnóstico ha facilitado la caracterización valorativa de los objetos de interés; aún se debe ahondar en la ponderación de estos valores mediante bases científicas, de manera que 

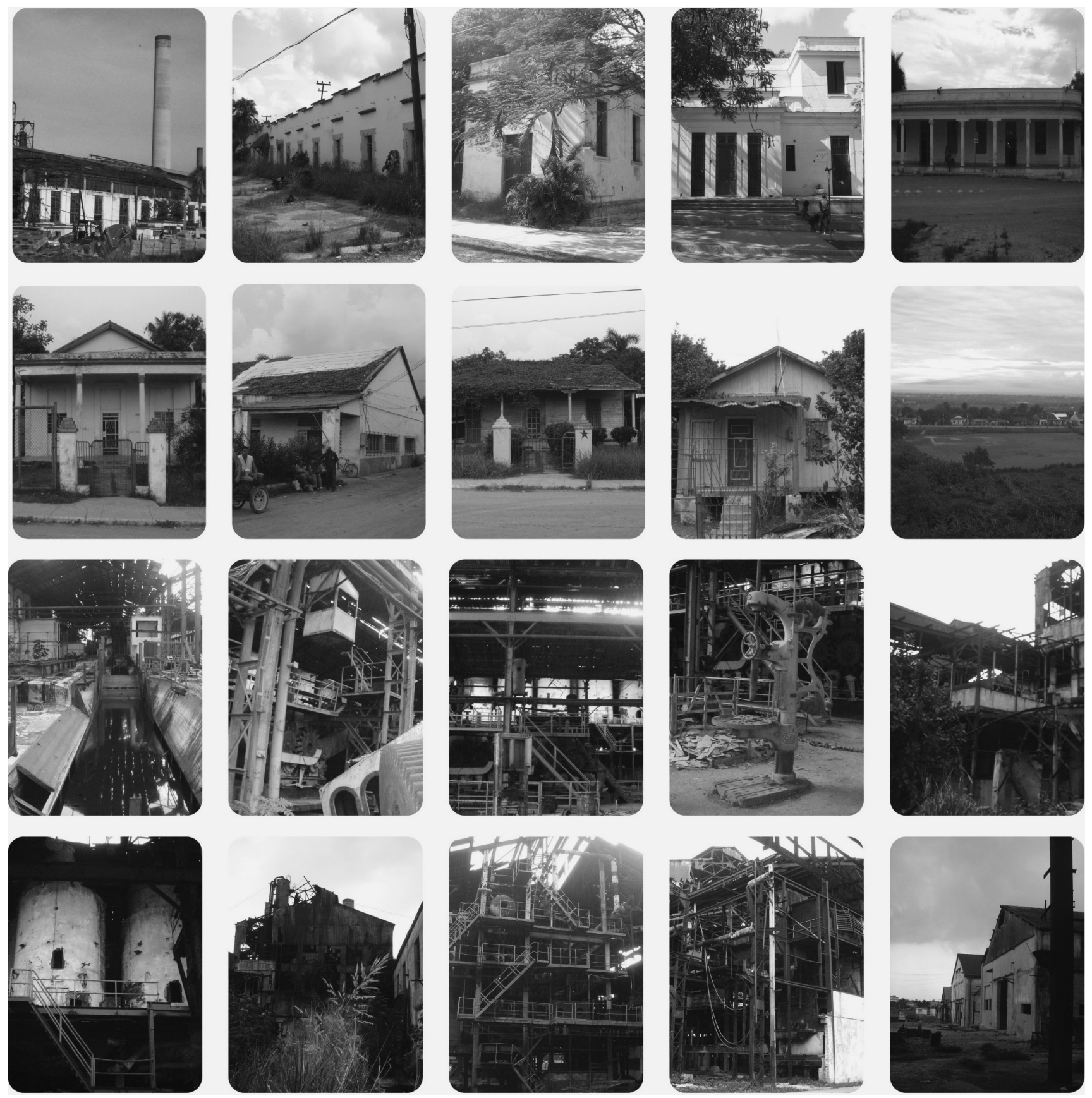

FIGURA 6. Elementos significativos del conjunto central Toledo (Fotografía: Adrián González González, Indira Costa Fallarero y Tania Gutiérrez Rodríguez, 2009).

sirvan para priorizar las acciones de intervención por realizar en cualquier propuesta de reutilización. Lo cierto es que esos elementos valorativos han de considerarse en el diseño de los potenciales usos a los que se destinen los espacios industriales en desuso y reciclados para que, de esta manera, cumplan con los actuales intereses productivos o sociales. Disímiles experiencias han probado que los conjuntos industriales tienen potencialidades para ser refuncionalizados y transformados, dependiendo de los condicionantes contextuales existentes y los escenarios futuros previsibles (cfr. Capel 1996:29). No obstante, no se debe olvidar que la principal potencialidad de estos conjuntos es la presencia de elementos con alto valor patrimonial, testigos de una cultura productiva heredada de siglos de desarrollo industrial azucarero en Cuba. Así, el patrimonio industrial azucarero puede convertirse en un elemento fundamental para acometer una transformación en el batey cubano con vistas a generar un nuevo desarrollo que satisfaga las necesidades de las comunidades de nuestro presente. 


\section{Referencias}

Barnet, Miguel 2005 "La cultura que generó el mundo del azúcar", Catauro: Revista Cubana de Antropología, 6 (11):6-15.

Capel, Horacio 1996 "La rehabilitación y el uso del patrimonio histórico industrial", Document d'Análisi Geográfica, 29:19-50.

Cárdenas, Eliana 1993 "Identidad: valores culturales, uso y significado", Arquitectura y Urbanismo, XIV (2):61-66. 1998 Problemas de teoría de la arquitectura, México, Universidad de Guanajuato (UG).

Cazanave Macías, Joiselen

2007 "Pautas metodológicas para el diseño de espacios de intercambio social accesibles por adultos mayores", tesis de doctorado en ciencias técnicas, Ciudad de La Habana, Facultad de Arquitectura, Instituto Superior Politécnico "José Antonio Echeverría" (CUJAE).

Charadán López, Fernando 1982 La industria azucarera en Cuba, La Habana, Editorial de Ciencias Sociales.

Costa Fallarero, Indira y Adrián González González

2009 "Toledo: valores, imagen y transformación", tesis de grado en arquitectura, Ciudad de La Habana, Cuba, Facultad de Arquitectura (CUJAE).

Cruz, Diana María

2009 "La vivienda en el batey azucarero del Oriente cubano", Arquitectura y Urbanismo, xxx (2):44-51.

Dembicz, A.

1989 Plantaciones cañeras, La Habana, Editorial de Ciencias Sociales.

Fernández Figueroa, Enrique Juan de Dios

2007 "Docencia y rehabilitación urbana: ciudad y arquitectura", Arquitectura y Urbanismo, XXVIII (3):56-60.

Gutiérrez Rodríguez, Tania

2008 "En Toledo, el azúcar da paso a otras formas de desarrollo", 14 Convención de Ingeniería y Arquitectura, La Habana, Cuba.

2013 "Azúcar y patrimonio en el central cubano", en Giovanni Luigi Fontana, J. Melgarejo y María Victoria Zardoya (eds.), Patrimonio hidráulico, industrial, arquitectónico y urbano en el ámbito hispano-cubano, Narni, Crace: 241-266.

Linares, María Teresa

2005 "Intervención en Seminario de la cultura del azúcar en Cuba", Catauro: Revista Cubana de Antropología, vı (11): 87.
López, Elmer y Jesús Sánchez

1982 Diseño básico, La Habana, Facultad de Arquitectura (CUJAE).

Lozano Zamora, Andrés

2009 El complejo cultural cubano del azúcar, Contribuciones a las Ciencias Sociales, documento electrónico disponible en [www.eumed.net/rev/cccss/06/alz.htm], consultado en junio de 2015.

Lynch, Kevin

1970 La imagen de la ciudad, La Habana, Instituto del Libro. ONU

1987 Informe de la Comisión Mundial sobre el Medio Ambiente y Desarrollo, Nuestro Futuro Común, documento mecanoescrito disponible en [http://www.un.org/es/comun/ docs/?symbol=A/42/427], consultado en diciembre de 2016. Ortiz, Fernando

1983 Contrapunteo cubano del tabaco y el azúcar, La Habana, Editorial de Ciencias Sociales.

Pell Delgado, Ilka

2013 "Rescate y conservación de las locomotoras a vapor", en Giovanni Luigi Fontana, J. Melgarejo y María Victoria Zardoya (eds.), Patrimonio hidráulico, industrial, arquitectónico y urbano en el ámbito hispano-cubano, Narni, Crace: 267-293.

Pereira, Ernesto

2009 "El batey de la industria azucarera en Villa Clara", Arquitectura y Urbanismo, xxx (2): 36-43.

Puig Jodar, Meritxell

2008 El patrimonio industrial en una sociedad cambiante, documento electrónico disponible en [http://www.ub.edu/ geocrit/-xcol/362.htm], consultado en mayo de 2015.

Rallo, Joaquín y Roberto Segre

s. f. Introducción histórica a las estructuras territoriales y urbanas de Cuba, 1519-1959, La Habana (CUJAE).

Ríos Díaz, Covadonga

2007 "Arquitectura industrial y posible reutilización, AI\&R", tesis de doctorado en ciencias técnicas, Oviedo, Escuela Técnica Superior de Ingenieros de Minas de Oviedo-Universidad de Oviedo.

$\mathrm{TICCIH}$

2003 Carta de Nizhny Tagil sobre el patrimonio industrial, 17 de julio de 2003, Moscú, International Committee for the Conservation of the Industrial Heritage, documento electrónico disponible en [www.icomos.org/18thapril/2006/nizhnytagil-charter-sp.pdf], consultado en octubre de 2016. 


\section{Síntesis curricular del/los autor/es}

\section{Adrián González González}

Facultad de Arquitectura

Universidad Tecnológica de la Habana "José Antonio Echeverría"

(CUJAE), Cuba

yoadrianxxi@gmail.com

Arquitecto (Instituto Superior Politécnico "José Antonio Echeverría" [CUJAE], Cuba). Profesor asistente del Departamento de Diseño (Facultad de Arquitectura-CUJAE). Miembro del grupo Investigación y Acción Urbanas (Invacurb).

\section{Indira Costa Fallarero}

Université Paris-Sorbone I, Francia

icostafall@gmail.com

Arquitecta (Universidad Tecnológica de la Habana "José Antonio Echeverría" [CUJAE], Cuba). Máster graduada del Programa
"Technique patrimoine et territoire de I'industrie", Université Paris-Sorbone I (Universidad París de la Sorbona I), Francia.

\section{Tania Gutiérrez Rodríguez}

Facultad de Arquitectura

Universidad Tecnológica de la Habana "José Antonio Echeverría"

(CUJAE), Cuba

taniarq1946@gmail.com

Arquitecta, doctora en ciencias técnicas (Bauhaus Universität Weimar, Alemania), profesora de mérito, titular y consultante de la Facultad de Arquitectura-CUJAE. Miembro del grupo Investigación y Acción Urbanas (Invacurb). Profesora invitada de la Universidad Federal de Roraima (Brasil).

Postulado/Submited 26.02.2016

Aceptado/Accepted 04.01.2017

Publicado/Published 31.01.2017

Ó 